\title{
Manifestations buccales
}

des maladies systémiques :

La maladie de Crohn

et la rectocolite hémorragique

\section{RÉSUMÉ}

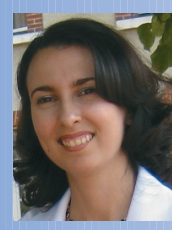

Samira CHERBI

Docteur en chirurgie dentaire Paris V, attachée hospitalo-universitaire

au Service d'odontologie de Bretonneau, $2^{\mathrm{e}}$ année de D.E.S.C.B.

\section{Claude-Bernard WIERZBA}

MCU-PH département de pathologie et chirurgie buccale

de l'université de Paris $\mathbf{V}$,

Service d'odontologie de Bretonneau.
Les entérocolites inflammatoires idiopathiques sont des pathologies systémiques d'étiologie inconnue et d'évolution chronique. Elles regroupent deux principales affections : la maladie de Crohn et la Rectocolite Hémorragique ( $\mathrm{RCH}$ ) dont les caractéristiques cliniques, topographiques et morphologiques sont nettement différentes. Ces pathologies peuvent être à l'origine de manifestations buccales qui précédent parfois de plusieurs années l'apparition des signes digestifs; d'où le rôle essentiel du chirurgien dentiste dans le diagnostic et la prise en charge précoce des patients souffrant de ces affections, d'autant plus qu'il existe un risque de dégénérescence maligne. De plus, en raison de la fréquence des récidives, ces patients bénéficient de traitements médicamenteux multiples pouvant poser un problème d'interaction avec nos prescriptions en odontologie.

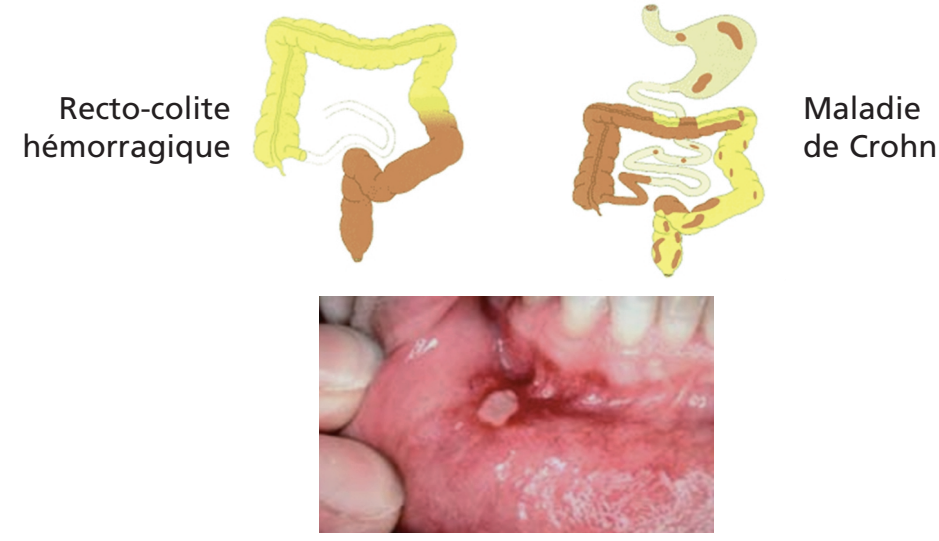

- pathologie inflammatoire

- maladie systémique

- lésions buccales

- origine idiopathique 


\section{La maladie de Crohn}

\section{$>$ Définition}

C'est une affection inflammatoire chronique d'étiologie inconnue pouvant atteindre tous les segments du tube digestif depuis la cavité buccale jusqu'à l'anus, mais le plus fréquemment l'iléon, le colon et l'anus; les lésions sont discontinues séparées par des zones de muqueuse saine.

\section{Épidémiologique}

C'est une affection qui prédomine dans les pays d'Europe et d'Amérique du Nord avec un gradient net nord-sud; elle atteint autant les hommes que les femmes avec un âge moyen de 20 à 30 ans[14, 20].

\section{Pathogénie}

La maladie de Crohn est une affection d'étiologie inconnue ; cependant, de nombreux facteurs de risque ont été identifiés parmi lesquels on peut citer :

\section{$>$ Les facteurs psychologiques}

Stress important, choc émotionnel (perte d'un proche), perte de l'estime de soi, états dépressifs.

\section{> Les facteurs génétiques}

Il existe une prédisposition familiale suggérée par la forte concordance entre jumeaux monozygotes. De plus des gènes de susceptibilité ont été identifiés (NOD2, CARD15) et une association à la spondylarthrite ankylosante est parfois retrouvée[10]. Des études récentes ont mis en évidence une association entre une mutation au niveau du gène codant pour le récepteur de I'IL23 et l'apparition de la maladie de Crohn ouvrant ainsi de nouvelles perspectives pour le dépistage et le traitement de cette pathologie[8].

\section{$>$ Les facteurs environnementaux}

Le tabagisme, le comportement alimentaire (régime riche en sucre et pauvre en fibres), la prise de médicaments (contraceptifs oraux), les agents infectieux pathogènes (mycobactéries, virus de la rougeole, listeriose...). Parmi ces facteurs, seul le rôle du tabac a été clairement identifié et il semblerait que ce soit plutôt la flore microbienne intestinale commensale (E. coli) qui serait à l'origine d'une réponse immunitaire inappropriée de l'hôte[10].

\section{> Les facteurs immunologiques}

L'action des bactéries commensales aboutit à une stimulation des lymphocytes $T$ helper et à la sécrétion de cytokines (IL1 et IL12) mais surtout du facteur tumoral (TNF $\alpha$ ). Ceci ayant pour conséquence des remaniements importants de la muqueuse intestinale[17].

\section{Anatomopathologie}

On observe au niveau de la muqueuse intestinale des lésions ulcéreuses avec une inflammation pariétale en dehors des zones ulcérées, un œdème du chorion et dans les lésions anciennes une fibrose de la sous-muqueuse.

Un élément est caractéristique de la pathologie retrouvé dans $50 \%$ des cas au niveau de la muqueuse intestinale et 67 à $85 \%$ des cas au niveau buccal[15] : «le granulome tuberculoïde non caséifiant» constitué de cellules épithélioïdes, de lymphocytes et de cellules géantes de Langhans (fig. 1). 


\section{Diagnostic positif}

\section{> Signes cliniques}

La maladie de Crohn est une pathologie chronique évoluant par poussées inflammatoires avec des symptômes digestifs et extra-digestifs :

\section{Des symptômes digestifs :}

- une diarrhée : le signe révélateur ;

- des douleurs abdominales le plus souvent localisées dans la fosse iliaque droite ;

- une masse abdominale palpable ;

- des sténoses digestives associées au syndrome de König (épisodes sub-occlusifs) ;

- des lésions anales : fissures, fistules et abcès péri-rectaux plus ou moins associés à des rectorragies ;

- un retentissement sur l'état général : fièvre, amaigrissement, asthénie ;

- complications possibles: occlusion du grêle suite à une sténose digestive, fistules, abcès, perforations et hémorragies voire une cancérisation.

\section{Les symptômes extra-digestifs :}

- articulaires : arthralgies, arthrites, spondylarthrite ankylosante, sacro- iléite ;

- oculaires : iritis, uvéite, épisclérite ;

- hépato-biliaires : stéatose, cholangite sclérosante, hépatite granulomateuse, cirrhose ;

- rénales : fistules, lithiases, amylose avec albuminurie élevée ;

- cutanées: érythème noueux (fig. 2), pyoderma gangrenosum, ulcérations cutanées granulomateuses ;

-thrombo-emboliques: hypercoagulabilité sanguine, thrombose du sinus caverneux.

\section{> Les manifestations bucco-dentaires}

\section{Des signes dentaires}

Plusieurs études mettent en évidence un risque accru de caries dentaires[7, 21].
De nombreux facteurs sont impliqués dans le processus de cariogenèse chez un patient atteint de la maladie de Crohn : le syndrome de malabsorption, un régime alimentaire riche en glucides et des repas fractionnés et répétés, une modification de certains composants salivaires en particulier lorsqu'il existe une granulomatose des glandes salivaires accessoires, une modification de la flore bactérienne avec une augmentation de Streptococcus mutans[19, 23].

\section{Les signes parodontaux}

Il existe une perte d'attache, une aggravation des poches parodontales[16], des récessions (fig. 3) et des mobilités dentaires qui semblent être liées aux phénomènes inflammatoires avec libération de cytokines (IL1, IL2) et de facteurs tumoraux (TNF $\alpha$ ) qui sont à l'origine de destructions tissulaires importantes. Cependant, ce lien de causalité n'a pas encore été clairement établi[10, 17].

\section{Les signes muqueux}

Ils sont retrouvés dans 4 à $14 \%$ des cas, plus fréquents dans la maladie de Crohn que dans la rectocolite ulcéro-hémorragique[17].

Ils peuvent parfois précéder de plusieurs années les manifestations gastro-intestinales et représenter ainsi le seul élément de diagnostic. Ces manifestations peuvent être soit liées à la maladie, soit y être associées.

\section{Les signes liés à la maladie de Crohn}

La maladie de Crohn fait partie d'un groupe très hétérogène d'affections qui s'accompagnent d'une inflammation granulomateuse de la muqueuse buccale que l'on appelle «la granulomatose orofaciale » GOF $[15,22]$ caractérisée par :

- des ulcérations linéaires: que I'on peut retrouver au fond du vestibule (le plus souvent) entourées de replis muqueux hyperplasiques et sont pathognomoniques de la maladie de Crohn. Elles peuvent également 
siéger au niveau du plancher buccal, sur la face interne des joues ou des lèvres (fig. 4) ;

- des lésions pavimenteuses: elles font suite aux ulcérations siégeant le plus souvent au niveau jugal ou labial ;

- des chéilites granulomateuses (fig. 5) : elles sont caractéristiques des granulomatoses orofaciales intéressant le plus souvent la lèvre inférieure ; l'œdème peut s'étendre au menton, à la joue voire à la paupière. Le diagnostic repose sur la biopsie de la lèvre qui permet de retrouver des "granulomes épithélioïdes et giganto-cellulaires non caséeux» (fig. 1).

\section{Les signes associés à la maladie}

- lésions ulcéreuses aphtoïdes récidivantes souvent confondues avec des aphtes récurrents (fig. 6) ;

- lichen plan ;

- pyostomatite végétante : affection cutanéomuqueuse rare considérée comme un marqueur des maladies inflammatoires intestinales. Elle est plus fréquente dans la rectocolite ulcéro-hémorragique. II s'agit d'une miliaire pustuleuse indolore qui atteint le plus souvent la muqueuse gingivale et labiale, respectant le plancher buccal formant par confluence un aspect caractéristique en «traces d'escargot» $[9,13]$ (fig. 7) ;

- le syndrome de Gougerot-Sjögren : modifications immunologiques des glandes salivaires accessoires[24] ;

- les dermatoses neutrophiliques (syndrome de Sweet)[13], le psoriasis et l'épidermolyse bulleuse acquise[9] ;

- les manifestations carentielles : carence en vitamines (B6, B12, C.) en magnésium, en zinc, à l'origine de lésions à type de glossites, d'agueusie et de stomatodynies ;

- plus rarement: des lésions vésiculo-pustuleuses[4], une dysgeusie métallique[12].

\section{> Signes biologiques}

- un syndrome inflammatoire : augmentation de la vitesse de sédimentation (VS) et de la protéine $C$ réactive $(C R P)$, une thrombocytose (supérieure à $400000 / \mathrm{mm}^{3}$ ) ;

- un syndrome de malabsorption : anémie et carence martiale, hypocholestérolémie et déficits vitaminiques (en particulier en folates et en vitamine B12);

- l'examen coprologique : à la recherche d'infections intestinales, il est surtout utile pour le diagnostic différentiel ;

- les examens sérologiques : avec le dosage des anticorps anti-Sacchromyces cerevisiae (ASCA) et des anticorps anti-cytoplasme des polynucléaires neutrophiles ou (PANCA) qui ont une bonne valeur prédictive mais ne sont pas spécifiques de la maladie de Crohn et de la rectocolite hémorragique; cependant, d'autres marqueurs ont été identifiés (anticorps antibactériens : anti-12, anti-Omp C) et sont en cours d'évaluation[10].

\section{> Signes radiologiques}

Différentes techniques d'imagerie permettent d'étayer le diagnostic :

- le cliché de l'abdomen sans préparation : utile dans les formes graves (pneumopéritoine) ;

- le transit du grêle et lavement baryté permettent de mettre en évidence des ulcérations, des sténoses, des pseudo-polypes (fig. 8 a) ;

- I'endoscopie avec biopsie : la coloscopie, examen indispensable au diagnostic mais aussi pour le pronostic, permet d'observer les lésions ulcéreuses, les sténoses et de réaliser des biopsies si des lésions suspectes sont retrouvées; il est ainsi utile pour le dépistage précoce des dysplasies (fig. 8 b) ;

- I'entéro-scanner et entéro-IRM : pour le bilan d'extension, il permet de rechercher d'éventuelles complications tel que les fistules, les abcès, les masses abdominales... 


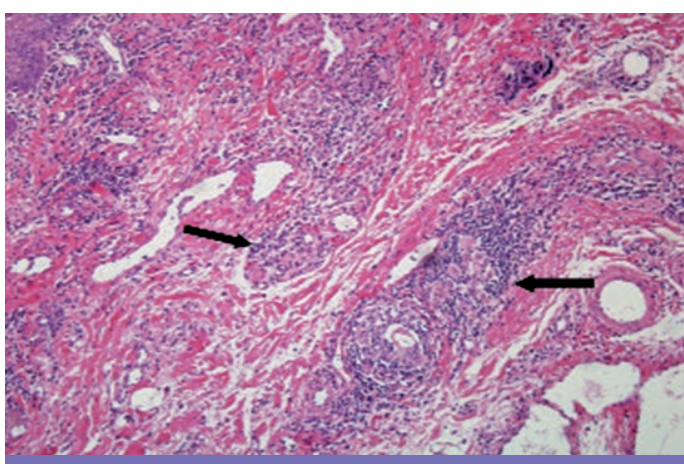

Fig. 1 Histologie: granulomes épithélioïdes et giganto-cellulaires non caséeux (flèches). On note aussi une légère dilatation des vaisseaux lymphatiques (hématoxyline et éosine, grossissement original x 10). JADC. A. Kauzman et al. 2006.

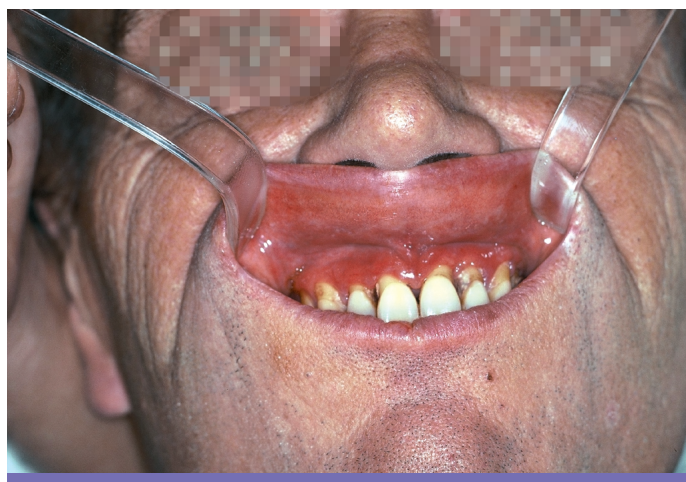

Fig. 3 Manifestations buccales: inflammation parodontale avec des récessions gingivales importantes. Dr C.B.Wierzba.

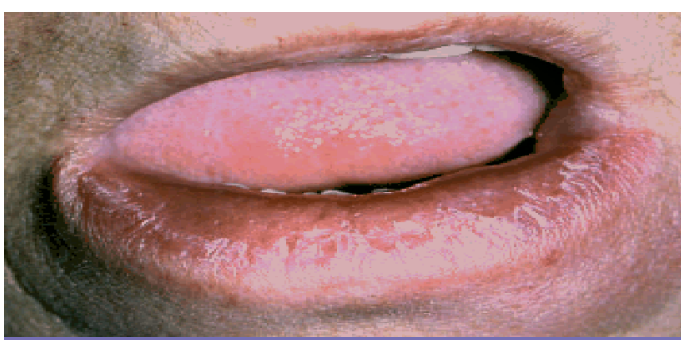

Fig. 5 Manifestations buccales: chéilite de la lèvre inférieure. On note une tuméfaction bilatérale et un érythème du menton. JADC. A. Kauzman et al. 2006.

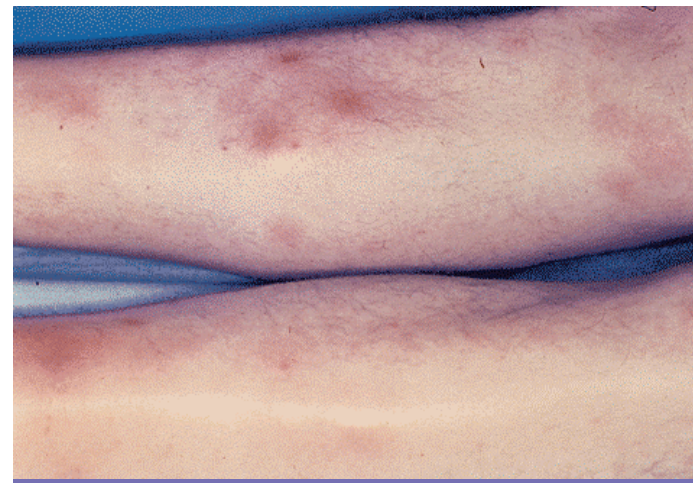

Fig. 2 Manifestations cutanées: l'érythème noueux avec des nodules érythémato-violacés situés sur la crête tibiale. HépatoGastro. J. Bonnet et al. 1999.

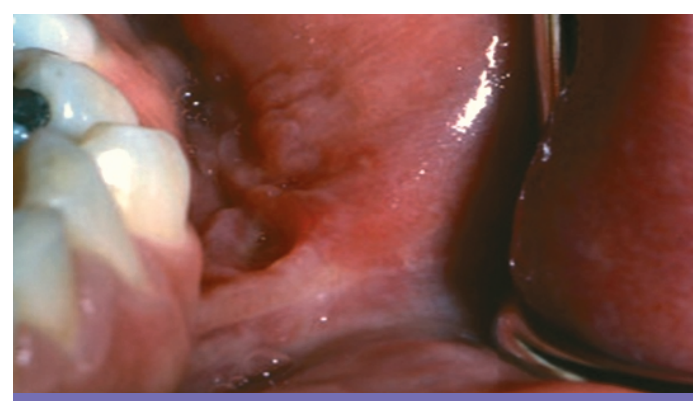

Fig. 4 Manifestations buccales : ulcère aphtoïde linéaire profond, entouré de replis de tissus hyperplasiques au fond du vestibule. JADC. A. Kauzman et al. 2006.

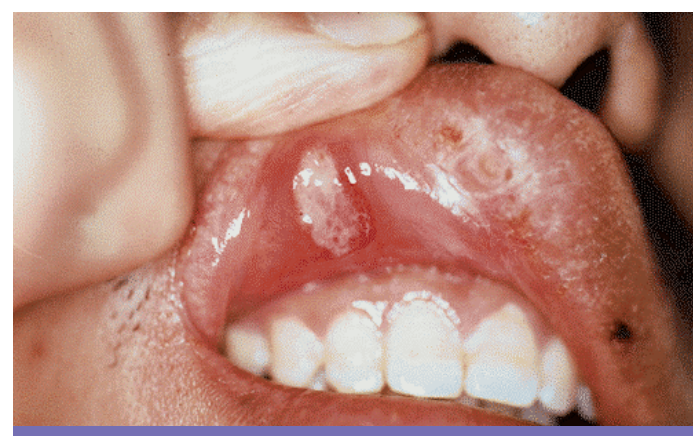

Fig. 6 Manifestations buccales: ulcération à fond déprimé, avec liseré rouge périphérique. Hépato-Gastro. J. Bonnet et al. 1999. 


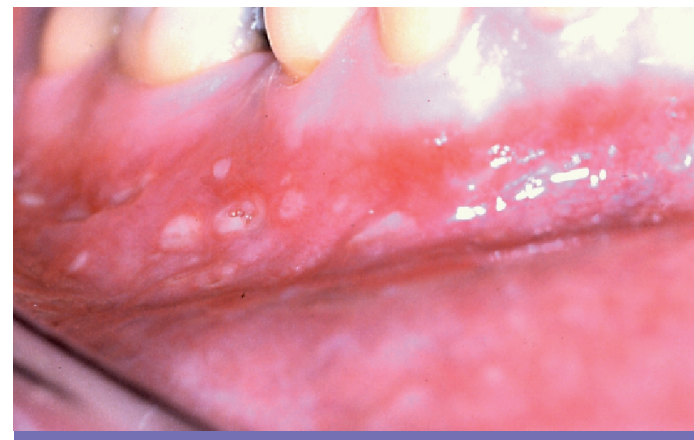

Fig. 7 Manifestations buccales: ulcérations au niveau du fond du vestibule avec des lésions pustuleuses en traces d'escargot. Dr Gilon Y. Service de chirurgie maxillofaciale, CHU Sart-Tilman, Liège.

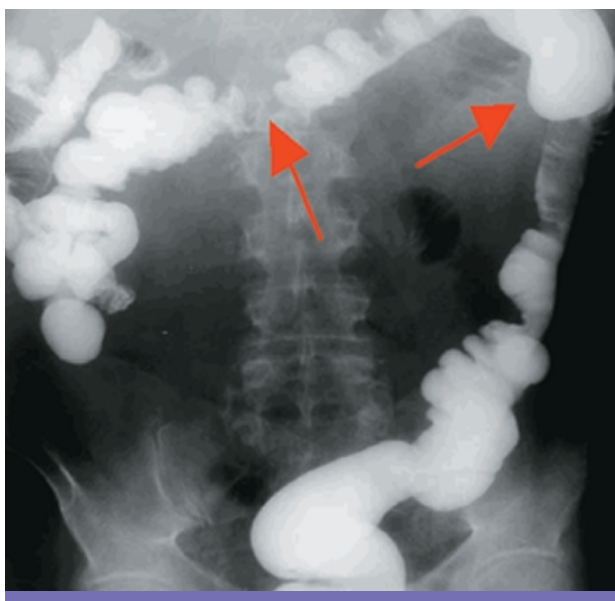

Fig. 8 a Lavement baryté. On Observe des sténoses et des dilatations (flèches). A. Oudjit et al. EMC Radiodiagnostic Appareil digestif 2005.

\section{Diagnostic différentiel}

\section{Pour les formes intestinales aigues}

- diarrhées aigues infectieuses: salmonelle, shigelle, amibiase giardiase ;

- appendicite ;

- infections à Yersinia enterocolitica.

\section{Dour les formes intestinales chroniques}

- les colites infectieuses : giardiases, amibiases, tuberculose intestinale ;

- lymphomes ;

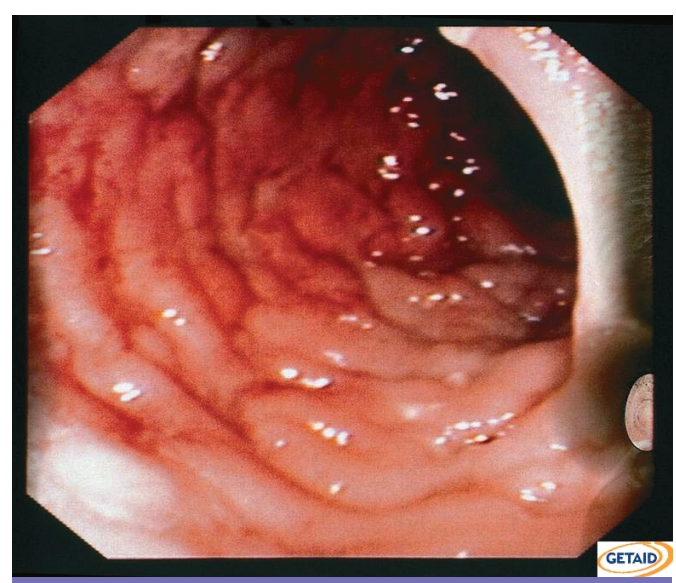

Fig. 8 b Endoscopie du colon montrant des lésions pavimenteuses de la muqueuse. www.getaid.org

- rectocolite hémorragique ;

- entérocolite pseudo-membraneuse ;

- colites ischémiques.

\section{Dour les formes buccales}

Les granulomatoses orofaciales: le syndrome de Melkersson-Rosenthal (SMR) et la chéilite granulomateuse (CG) de Miescher, la sarcoïdose ou, plus rarement, la maladie de Wegener.

La maladie de Behçet (pour les formes avec lésions aphteuses). 


\section{La rectocolite hémorragique}

\section{Définition}

C'est une pathologie inflammatoire chronique idiopathique, touchant le rectum voir le colon gauche, respectant le grêle et l'anus. Elle se manifeste essentiellement par des diarrhées hémorragiques. L'atteinte est toujours continue sans intervalle de muqueuse saine.

\section{Épidémiologie}

Fréquence plus élevée dans les pays industrialisés.

L'âge de survenue : il existe 2 pics : $15-35$ ans et 55-65 ans.

II n'existe pas de différence significative entre les deux sexes[14, 20].

\section{Pathogénie}

Bien que la rectocolite hémorragique soit une affection idiopathique, de nombreux facteurs semblent intervenir dans la pathogenèse de cette maladie.

\section{> Les facteurs génétiques}

Les patients porteurs du système majeur d'histocompatibilité (HLA) type HLA-DRB1 et DQB1 semblent être plus susceptibles de développer une colite ulcéreuse. Il existe donc une notion de prédisposition familiale[11].

\section{> Les facteurs environnementaux}

- le tabagisme : dont le rôle est moins bien établi que dans la maladie de Crohn ;

- les contraceptifs oraux ;

- les régimes alimentaires pauvres en fibres ;

- un niveau socio-économique élevé ;

- les agents microbiens : rougeole, mycobactéries, listériose...

\section{> Les facteurs psychogènes}

Il existe une composante psychique et émotionnelle (surmenage, stress, perte d'un proche, dépression...) qui peut être à l'origine du déclenchement ou d'une rechute de la maladie.

\section{> Les facteurs immunologiques}

Le mécanisme est similaire à celui de la maladie de Crohn avec libération de cytokines (IL1, IL2, IL10) et de facteurs tumoraux (TNF $\alpha$ ).

\section{Anatomopathologie}

Sur le plan macroscopique, on observe une dilatation ou une sténose colique ; la muqueuse est rouge congestive saignant au contact. Les lésions sont diffuses du rectum au colon gauche sans intervalle de muqueuse saine.

Sur le plan histologique : l'épithélium subit un décollement favorisant l'apparition d'ulcérations, le chorion est œdèmatié et congestif. II existe également des anomalies glandulaires (abcès cryptiques). Dans les formes chroniques, on peut observer une fibrose de la sousmuqueuse.

\section{Diagnostic positif}

\section{$>$ Les signes cliniques}

La rectocolite hémorragique étant une maladie systémique, elle se manifeste par des signes digestifs et extra-digestifs :

\section{Les signes digestifs :}

Le début peut être brutal ou insidieux. II existe parfois des facteurs déclenchants (surmenage, perte d'un proche, antibiothérapie...). 
Le symptôme dominant est la diarrhée le plus souvent hémorragique avec des émissions rectales de sang (rectorragies), de pus ou de glaires associés à des ténesmes.

Le patient peut se plaindre également de douleurs abdominales localisées à la fosse iliaque gauche ou diffuses à tout l'abdomen avec parfois des douleurs anales.

Un retentissement sur l'état général est fréquent en particulier lors des poussées : fièvre, amaigrissement, asthénie, anorexie, nausées et vomissements.

Des complications peuvent survenir au cours de l'évolution de la maladie parmi lesquelles on peut citer: le mégacolon toxique (distension importante du colon), perforations, hémorragies massives, septicémies, complications thromboemboliques, cancérisation (18 à $20 \%$ de risque à partir de 10 à 20 ans d'évolution)[11, 22].

\section{Des signes extra-digestifs :}

- articulaires : la polyarthrite périphérique, la sacro-iléite et la spondylarthrite ankylosante ;

- oculaires : l'uvéite, l'épisclérite ;

- hépato-biliaires: la stéatose, la cholangite sclérosante, I'hépatite, voir la cirrhose ;

- hématologique et vasculaire : anémie hémolytique auto-immune, purpura auto-immun, thromboses veineuses ;

- pancréatique : la pancréatite chronique ;

- rénales : la lithiase rénale, l'amylose ;

- cardio-pulmonaires : les broncho-pneumopathies chroniques, la sarcoïdose, péricardite, endocardite ;

- endocriniennes : I'hyperthyroïdie ;

- cutanées : l'érythème noueux et le pyoderma gangrénusum.

\section{> Les manifestations buccales}

- I'aphtose buccale : moins fréquente que dans la maldie de Crohn (fig. 9) ;
- les lésions ulcéreuses de gravité et d'étendue variables en fonction de la gravité de la pathologie ;

- la pyostomatite végétante: manifestation buccale retrouvée dans les maladies inflammatoires intestinales en particulier dans la rectocolite. Elle se traduit par des lésions ulcéreuses et pustuleuses intéressant le palais et la muqueuse labiale. Elle peut être associée à une dermatose : la pyodermite végétante qui atteint la région axillaire et vulvopérinéale (fig. 10) ;

- l'épidermolyse bulleuse acquise qui évolue indépendamment des manifestations digestives; le diagnostic repose sur l'examen histologique avec immuno-fluorescence directe qui met en évidence des dépôts d'lgG et de C3 sous la lamina densa ;

- les manifestations buccales carentielles: glossite, chéilite, glossodynies, stomatodynies (fig. 11).

\section{$>$ Les signes biologiques}

Ils sont liés à la déshydratation et aux pertes électrolytiques par diarrhées profuses et sanglantes: anémie microcytaire et hyposidérémique, hématocrite abaissée, hypokaliémie, hypocalcémie et hypomagnésémie mais aussi à I'inflammation chronique : augmentation de la VS et de la CRP et hypoalbuminémie. Ces paramètres sont d'autant plus altérés que le stade de la maladie est avancé.

\section{> Les signes radiologiques}

- radiographie de l'abdomen sans préparation (ASP) :

- le lavement baryté (fig. 12 a) ;

- l'endoscopie : rectosigmoïdoscopie et coloscopie, permettant une visualisation directe des lésions avec possibilité de réaliser des biopsies si des lésions suspectes sont retrouvées (fig. 12 b) ;

- la tomodensitométrie : pour le bilan d'extension. 

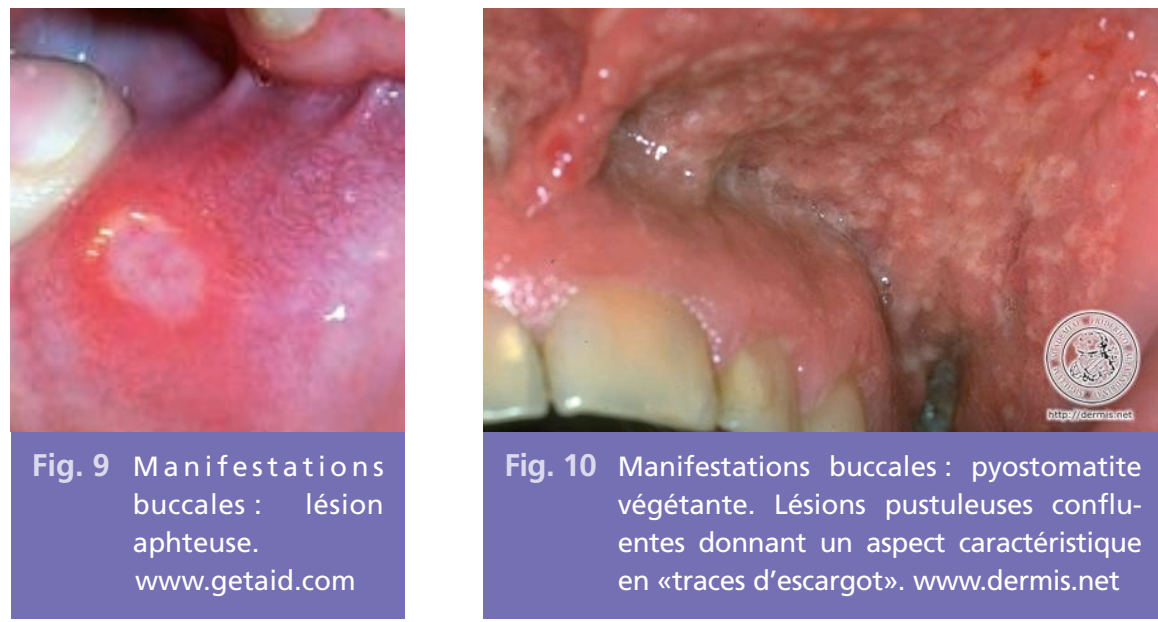

Fig. 10 Manifestations buccales: pyostomatite végétante. Lésions pustuleuses confluentes donnant un aspect caractéristique en «traces d'escargot». www.dermis.net

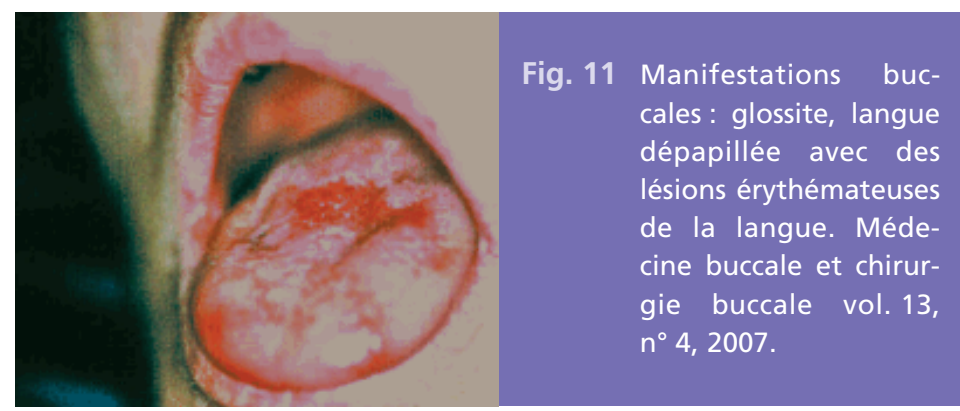

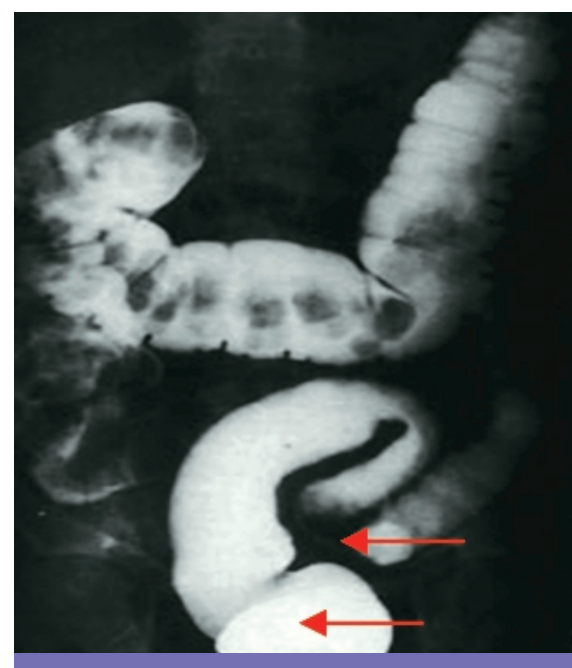

Fig. 12 a Lavement baryté disparition des haustrations et dilatation recto-sigmoïdienne avec sténose (flèches). K. Farahat et al. EMC gastro-entérologie, 1999.

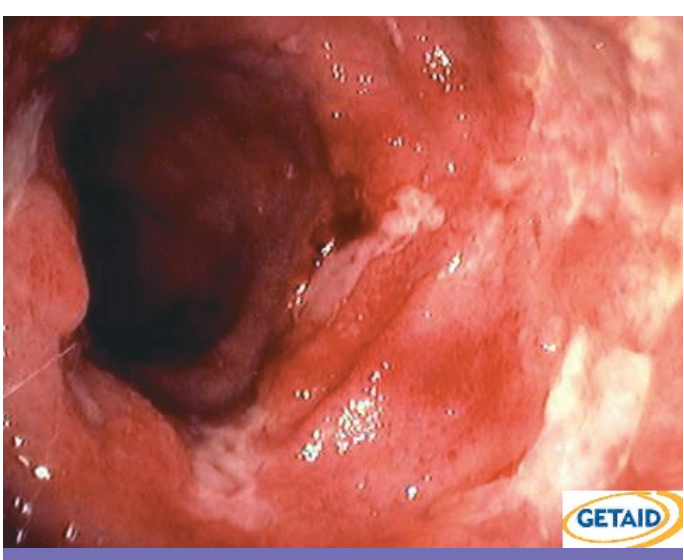

Fig. 12 b Endoscopie : aspect hémorragique avec ulcérations. www.getaid.org. 


\section{Diagnostic différentiel}

- la maladie de Crohn ;

- les colites infectieuses (shigella, salmonella, giardiase...) ;
- les colites ischémiques ;

- les colites pseudo-membraneuses ;

- le syndrome du colon irritable ;

- la maladie de Behçet (dans les formes avec aphtes).

\section{Le traitement de la maladie de Crohn et de la RCH}

\section{Les mesures \\ hygiéno-diététiques}

Régime sans résidus, en évitant les aliments stimulant le transit intestinal (alcool, café, agrumes...).

Correction de la malnutrition, de la déshydratation et de l'anémie.

Repos au lit pendant les phases actives.

\section{Le traitement médical}

Il est plus actif et plus efficace sur la rectocolite que sur la maladie de Crohn :

\section{> Les anti-inflammatoires intestinaux}

Il s'agit d'amino-salicylés avec deux molécules principales :

- la sulfasalazine : SALAZOPYRINE ${ }^{\circledR}$;

- les dérivés de l'acide 5-aminosalicylique (5ASA) ou mesalazine : PENTASA ${ }^{\circledR}$, ROWAZA ${ }^{\circledR}$ et Olsalazine : DIPENTUM ${ }^{\circledR}$.

Ils sont administrés par voie orale ou rectale. Les effets secondaires des salicylés sont :

La sulfasalazine : composée de 5-ASA et d'un sulfamide : nausées, vomissements, carence en acide folique, syndrome de Lyell, anémie hémolytique, neutropénie, thrombopénie. Plus rarement une agranulocytose, une pancréatite, une hépatite, une péricardite.

La mesalazine: composée uniquement de 5ASA : les effets secondaires sont analogues aux précédents mais moins fréquents.
L'olsalazine: une diarrhée peut parfois être observée.

\section{> Les corticoïdes}

Efficaces uniquement lors des poussées, ils sont utilisés par voie orale et rectale.

Il s'agit de la prednisone : CORTANCYL $^{\circledR}$ et de la prednisolone : SOLUPRED ${ }^{\circledR}$ mais le budésonide : ENTOCORT ${ }^{\circledR}$ à libération contrôlée iléocolique constitue maintenant un traitement de première intention dans les poussées d'intensité légère à modérée.

Les effets indésirables d'un traitement corticoïde au long cours sont : les troubles hydroélectrolytiques, l'ostéoporose, l'ostéonécrose aseptique, le retard de cicatrisation, l'immunosuppression, I'ulcère gastro-duodénal, les hémorragies digestives et troubles neuropsychiatriques.

\section{$>$ Les immunosuppresseurs}

En raison de la composante immunologique de ces pathologies, un traitement immunosuppresseur est possible avec plusieurs molécules: - I'azathioprine: IMUREL ${ }^{\circledR}$, la 6-mercaptopurine : PURINETHOL ${ }^{\circledR}$ ont des propriétés cytotoxiques et immunosuppressives. Leurs effets indésirables sont: une toxicité hématologique (leucopénie voire thrombopénie), hépatite choléstatique, pancréatite, diarrhées et vomissements ;

- le méthotrexate : MÉTHOTREXATE BELLON ${ }^{\circledR}$, NOVATREX ${ }^{\circledR}$ inhibiteur de la dihydrofolate 
réductase, a un effet cytotoxique et antiinflammatoire, il est surtout indiqué dans la maladie de Crohn; les effets indésirables sont : nausées, vomissements, diarrhées, ulcérations buccales et mucites, toxicité hématologique (leucopénie, thrombopénie voir pancytopénie), hépato-toxicité. Ces effets sont atténués par la prescription d'acide folique ;

- la cyclosporine : SANDIMMUN ${ }^{\circledR}$, NÉORAL $^{\circledR}$ agit par inhibition de la signalisation par I'IL-2 sécrétée par les précurseurs des T-helpers, inhibant ainsi la formation des lymphocytes $T$ killers et donc la cytolyse tissulaire. Elle est indiquée dans le traitement de la rectocolite. Les effets indésirables sont: nausées, vomissements, une néphrotoxicité, l'hépato-toxicité, une hyperuricémie, I'hypertension artérielle, l'hyperplasie gingivale, les infections[3].

\section{$>$ Autres molécules}

Anticorps anti-TNF $\alpha$ : Infliximab : REMICADE ${ }^{\circledR}$ c'est un inhibiteur de l'activité du TNF $\alpha$. II est utilisé en particulier pour la maladie de Crohn à forme luminale et fistulisante ou associé à une pyostomatite vegétante[6].

L'antibiothérapie : métronidazole : FLAGYL ${ }^{\circledR}$

Le cromoglycate de sodium.

Le Thalidomide pour le traitement de I'aphtose buccale (avec précaution en raison du risque tératogène)[22].

\section{Prise en charge psychologique}

La maladie de Crohn et la rectocolite sont des pathologies à composante psychologique. De plus, la chronicité, la fréquence des rechutes et les handicaps post-opératoires fragilisent les patients sur le plan psychologique avec un retentissement familial et socio-professionnel important.

Une prise en charge médicamenteuse (antidépresseurs, anxiolytiques...) avec une psychothérapie sont souvent nécessaires.

\section{Le traitement chirurgical}

II est indiqué en cas d'échec du traitement médical.

Dans la RCUH, 20 à $25 \%$ des patients nécessiteront, au décours de l'évolution de leur maladie, une colectomie[3,22]. Elle est souvent associée à une iléostomie conventionnelle voire à une anastomose iléorectale[11].

Dans la maladie de Crohn, la chirurgie est moins favorable, cependant, une procto-colectomie avec iléostomie peut être réalisée et induit une fréquence de rechutes de l'ordre de $50 \%$ au cours des 10 années suivant l'intervention[22].

\section{La place de l'odontologiste dans la prise en charge des patients}

\section{Sur le plan diagnostique}

Le chirurgien-dentiste représente parfois la première personne qui découvre et diagnostique une maladie de Crohn ou une rectocolite ulcéreuse, les manifestations buccales pouvant précéder de plusieurs années les signes intestinaux $[16,17]$.

Il est donc nécessaire de savoir reconnaître ces signes lorsqu'ils existent afin d'adresser le patient à un spécialiste en gastro-entérologie ou en médecine interne, des examens et des 
explorations spécifiques à ces affections pourront être ainsi réalisés pour affiner le diagnostic.

II faudrait donc former les odontologistes à I'identification de ces lésions, d'autant plus que ces affections peuvent parfois avoir un pronostic péjoratif (existence d'un risque de dégénérescence maligne des lésions digestives).

\section{Pour le traitement des manifestations buccales}

Les lésions buccales répondent le plus souvent au traitement de fond de la maladie de Crohn et de la rectocolite, en particulier avec les corticoïdes. Cependant, certaines lésions nécessitent un traitement spécifique :

- I'aphtose buccale : traitement local à l'acide acétylsalicylique en bains de bouche en première intention à visée antalgique et antiinflammatoire, voire xylocaïne gel visqueux $5 \%$ (six à huit applications par jour), le sucralfate ou ULCAR ${ }^{\circledR}$ en bains de bouche pur ou légèrement dilué; les stéroïdes locaux sous forme de béthaméthasone en tablettes (BETNÉVAL ${ }^{\circledR}$ ) 2 fois $5 \mathrm{mg} / \mathrm{j}$.

Les traitements généraux sont indiqués en seconde intention et dans les formes sévères: COLCHICINE ${ }^{\circledR} 1 \mathrm{mg} / \mathrm{j}$ pendant 3 mois, avec adaptation des doses par la suite; DISULONE ${ }^{\circledR} 100 \mathrm{mg} / \mathrm{j}$ (après dosage de l'activité G-6-PD et sous surveillance); Thalidomide $100 \mathrm{mg} / \mathrm{j}$; corticothérapie générale de courte durée[3, 11].

- la pyostomatite végétante dans les formes cortico-résistantes: I'administration de l'infliximab puis d'une cure de methotrexate permet d'obtenir de meilleurs résultats[6] ;

- l'hyperplasie gingivale : en plus de la motivation à l'hygiène avec détartrage et surfaçage, une excision chirurgicale peut être réalisée (gingivectomie). Cependant, les récidives sont fréquentes nécessitant plusieurs réinterventions[1].

\section{Pour les soins dentaires et les prescriptions}

Ces patients sont à risque carieux et parodontal majoré, ils devront donc être sensibilisés et faire l'objet d'une grande attention concernant leur hygiène buccale.

Les chirurgies invasives devront être évitées en période de crises. De même que la mise en place d'implants est déconseillée (en raison de l'ostéoporose associée à ces pathologies et secondaire au traitement par corticoïdes ainsi qu'au risque infectieux majoré par les médicaments immuno-suppresseurs) $[2,5]$.

Pour les soins dentaires et en particulier l'anesthésie, il ne semble pas exister de contre-indications aussi bien en local qu'en anesthésie générale[22].

\section{$>$ Ce sont des patients poly-médicamentés}

Concernant les salicylés : en raison du risque de thrombopénie et de leucopénie il existe un risque hémorragique et infectieux ; une antibiothérapie et des moyens d'hémostase locale sont donc nécessaires pour les gestes invasifs. De plus, I'utilisation d'anti-inflammatoires non stéroïdiens comme antalgiques (en particulier les salicylés) doivent être évités.

Les corticoïdes : I'utilisation d'anti-inflammatoires non stéroïdiens est proscrite en raison du risque majoré d'ulcère gastro-duodénal. De même, leur effet immuno-suppresseur nécessite le recours à l'antibiothérapie pour les soins invasifs en évitant l'association amoxicillineacide clavulanique qui peut aggraver une diarrhée pré-existante. 
Les immuno-suppresseurs: à l'origine d'un risque infectieux et hémorragique nécessitant des précautions particulières (hémostase locale et antibiotiques).

Les médicaments à l'origine de manifestations buccales :

- les anti-dépresseurs et anxiolytiques: on observe une diminution de la sécrétion salivaire et donc un risque carieux élevé ;

\section{Conclusion}

Le chirurgien dentiste joue un rôle essentiel dans le dépistage des maladies inflammatoires chroniques intestinales ( $\mathrm{MICl})$. Il permet grâce aux soins bucco-dentaires d'améliorer le confort oral du patient, élément déterminant dans sa qualité de vie (d'autant plus qu'il existe
- la cyclosporine qui induit une hyperplasie gingivale récidivante, très difficile à traiter ;

- les immuno-suppresseurs qui peuvent induire des candidoses buccales résistantes aux traitements anti-fongiques.

Dans tous les cas, il est indispensable de prendre contact avec le médecin traitant afin de discuter des modalités de traitement et des prescriptions post-opératoires.

souvent un problème de dénutrition). Une prise en charge multidisciplinaire serait donc souhaitable en collaboration avec les médecins spécialistes afin d'établir une stratégie thérapeutique adaptée au patient et à sa pathologie.

Tableau comparatif : maladie de Crohn vs RCH.

\begin{tabular}{|c|c|c|}
\hline & Crohn & $\mathbf{R C H}$ \\
\hline Âge de survenue : & Sujet d'âge moyen & Sujet jeune \\
\hline Topographie des lésions & $\begin{array}{l}\text { Discontinues : } \\
\text { iléon +++, } \\
\text { colon, anus }\end{array}$ & $\begin{array}{l}\text { Continues: } \\
\text { colon et rectum }\end{array}$ \\
\hline Atteinte rectale : & Inconstante & Constante \\
\hline Diarrhées: & ++ & ++ \\
\hline Rectorragies : & + & ++ \\
\hline Signes extradigestifs : & + & + \\
\hline Pyostomatie végétans : & $+/-$ & + \\
\hline $\begin{array}{l}\text { Risque } \\
\text { de dégénérescence } \\
\text { maligne: }\end{array}$ & $+/-$ & + \\
\hline
\end{tabular}




\section{Bibliographie}

1. Abjean J,

Bonnaure-Mallet $\mathrm{M}$,

Letoux G.

Étiopathogenie

et traitement

des hyperplasies gingivales

des malades

sous cyclosporine.

A Rev Odontostomatol 1990;19(5):423-429.

2. Alsaadi G, Quirynen M, Komárek A,

Van Steenberghe D. Impact of local and systemic factors on the incidence of failures up to abutment connection with modified surface oral implants.

Journal of Clinical

Periodontology

2007;34(7):610-617.

3. Astier A.

Traitement de la rectocolite ulcéro-hémorragique et de la maladie de Crohn. In Pharmacie Clinique et thérapeutique.

Association des Enseignants de Pharmacie Clinique Ed. $2^{\mathrm{e}}$ ed: Masson, Paris 2002:229-247.

4. Attar A, Laudenbach $P$, Jabbour $M$ et coll.

Lésions buccales

et cutanées

au cours de la maladie de Crohn.

A propos d'une observation. Rev Stomatol Chir Maxillofac 1985;86:382-385.

5. Balshi TJ, Wolfinger GJ. Management of the posterior maxilla in the compromised patient: historical, current, and future perspectives. Periodontology 2000;33:67-81.
6. Bens G, Laharie D, Beylot-Barry M et coll. Successful treatment with infliximab and methotrexate of pyostomatis vegetans associated with Crohn's disease. British J of Dermatol 2003;149:181-184.

7. Bevenius J.

Caries risk in patients with Crohn's disease: a pilot study. Oral Surg Med Pathol 1988;65:304-307.

8. Duerr $\mathrm{RH}$, Taylor $\mathrm{KB}$, Brant SR, Cho JH et coll.

A Genome-Wide Association Study Identifies IL23R as an Inflammatory Bowel Disease Gene. Science online, octobre 2006.

9. Delaporte $\mathrm{E}$, Viget $\mathrm{N}$, Pasturel-Michon $U$ et coll. Pyostomatite-pyodermite végétante révélatrice d'une maladie de Crohn. Ann Dermatol Venereol 1998;125:331-334.

10. De Saussure $P$, Bouhnik $Y$. Maladie de Crohn. Encycl Med Chir (Elsevier, Paris), Gastro-entérologie, 9-057-G-10, 2007, 18 p.

11. Farahat $K$, Sobhani $I$, Bonnaud G, Vallot T, Vissuzaine $C$, Mignon $M$. Rectocolite ulcérohémorragique : épidémiologie, physio-pathologie, diagnostic, histoire naturelle et stratégie thérapeutique. Encycl Med Chir
(Elsevier, Paris),

Gastro-entérologie, 9-059-A-10, 1999, 24 p.

12. Franckel $\mathrm{DH}$, Mostifi, Lorincz AL.

Oral Crohn's disease: report of two cases in brothers with metallic dysgueusia and review of literature. J Am Acad Dermatol 1985;12:260-268.

13. Guerre-Schmidt $A R$, Pelletier F, Carbonnel F et coll.

Syndrome arthrocutané associé à une maladie de Crohn chez une adolescente. La Revue de Médecine Interne 2006;27:874-877.

14. Harrison TR.

Principes

de médecine interne.

5e ed 1995.

Médecine-Sciences Flammarion;1269-1281.

15. Kauzman A,

Quesnel-Mercier A, Lalonde B.

Orofacial granulomatosis:

2 case reports and literature review.

J Can Dent Assoc 2006;72(4):325-329.

16. Lazaro $\mathrm{P}$. Réhabilitation buccale chez un sujet atteint de maladie de Crohn. Chir Dent Fr 1990;544:57-64.

17. May K

La maladie de Crohn : incidences odonto-stomatologiques. Thèse de $2^{\mathrm{e}}$ cycle, doctorat en chirurgie dentaire. Université de Nantes, 1999. 
18. Monteil RA, Fafet A, Raybaud H, Limouse L. La maladie de Crohn : un piège pour le praticien, des soucis pour le patient. Le chirurgien dentiste de France Fev 2001;1017:29-31.

19. Muerman et coll. Gingival and dental status, salivary acidogenic bacteria and yeast counts of patients with active or inactive Crohn's disease. Oral surg Med Pathol 1994;77:465-468.
20. Paitra-Cohen $S$, Halphen M,

Bouché $O$.

Diarrhées chroniques.

Impact internat

hépato-gatro-entérologie 1994;6:81-97.

21. Rooney TP.

Dental caries

prevalence

in patients

with Crohn's disease.

Oral Surg Med Pathol 1984;57:623-624.

22. Scully C, Cawson RA. Medical problems in dentistry. Elsevier, 5e edition, 2005.
23. Sundh B, Emilson CG.

Salivary

and microbial conditions

and dental health

in patients

with Crohn's disease:

a 3 year study.

Oral Surg Med Pathol

1989;67:286-290.

24. Triantafillidis JK,

Roussou P,

Manousoson et coll.

Ulcerative colitis and Sjogren's syndrome in the same patient: report of two cases and review of literature. Ital J Gastroenterol 1994;26:299-302.

\section{SUMMARY}

\section{Oral symptoms of systemic pathologies: Crohn's disease and ulcerative colitis}

Samira CHERBI

Claude-Bernard WIERZBA

\section{Keywords}

- inflammatory bowel disease

- oral symptoms

- systemic pathologies
Inflammatory bowel disease (IBD) are systemic pathologies with chronic disorders, and originate from unidentified causes. Two main types exist: Crohn's disease and ulcerative colitis, both of which have very different clinical, topographic and morphological characteristics. Because the oral symptoms for these diseases can appear several years before digestive signs, the odontologist have a critical role in early diagnosis and patient treatment, all the more so than cancer hazards can be involved. Furthermore, as the treatments of this type of pathologies is complex and involves different kinds of medication, it may lead to interaction problems with odontologist prescriptions. 


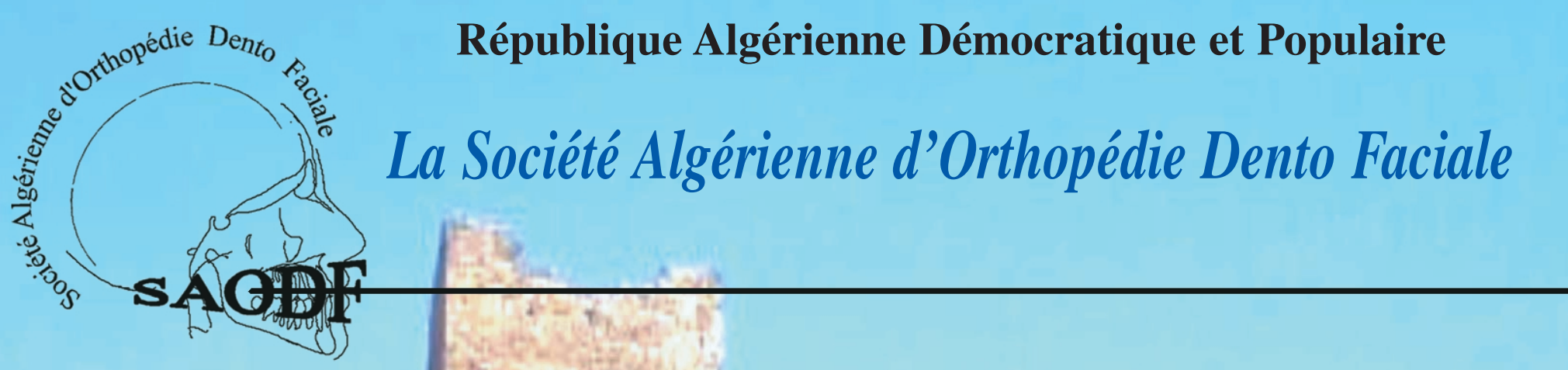

EN PARTENARIAT AVEC

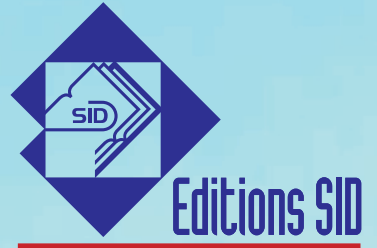

Groupe EDP Sciences www.editionsid.com

67) Sous le Haut Patronage du Wali de la Wilaya de Tlemcen

Les 26 et 27 Septembre 2009

A l'auditorium de la faculté de Tlemcen Son Congrès Inaugural souls le thème

DE L'ORTHOPÉDIE À L'ORMHODONAIE

ORGANISE

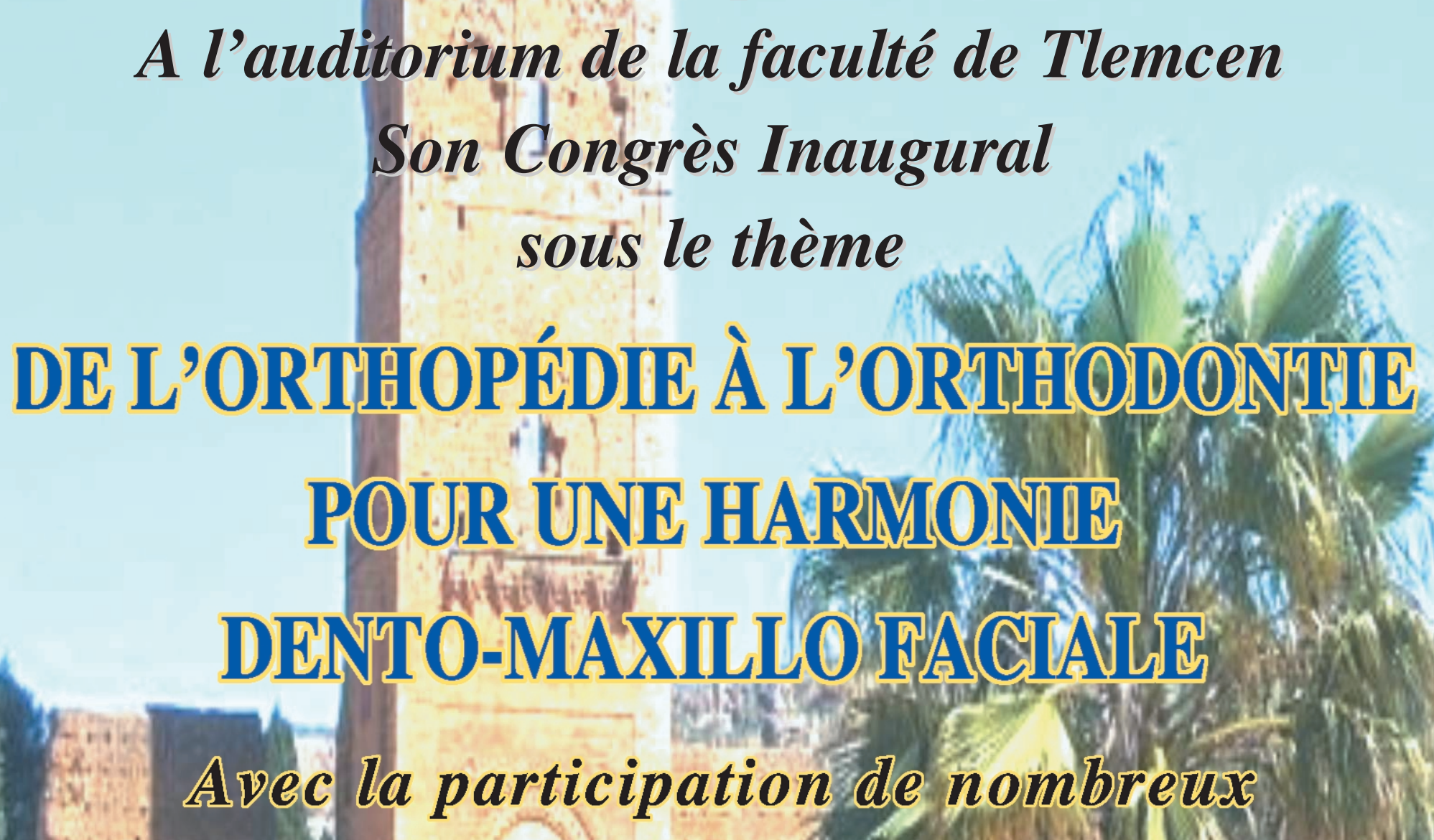

Orthodontistes Nationaux êt Interpationaux HSiege SAODF : 14, Boulevaral des 5 Martyrs Tlemsend Algerie 13000

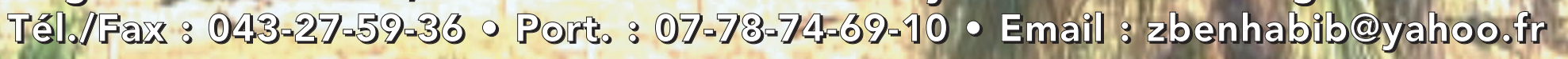

